\title{
The application example of the sensitivity analysis of the solution to coefficients of the $k-\varepsilon$ model
}

\author{
Ewa Błazik-Borowa \\ Department of Structural Mechanics, Faculty of Civil Engineering and Architecture, \\ Lublin University of Technology, e-mail: e.blazik@pollub.pl
}

\begin{abstract}
The paper presents the dependence between the degree of approximation of flow property functions and the sensitivity coefficients which are results of sensitivity analysis of solution to the coefficients of the $k-\varepsilon$ turbulence model. It is shown that the model coefficients are factors of derivatives in the set of equations which describes the $k-\varepsilon$ turbulence model. Because of this fact, when the term with the given parameter is large enough then the value of this parameter, being only the factor, has also larger influence on the solution. The numerical research is preceded by the description of calculation of sensitivity coefficients and their physical sense. The problem is presented for three tasks of incompressible steady flow around square and rectangular models with use of the standard and RNG versions of the $k-\varepsilon$ turbulence model.
\end{abstract}

Keywords: coefficients of the $k-\varepsilon$ turbulence model, sensitivity analysis, twodimensional flow around square and rectangular cylinders.

Nomenclature:

$C_{\varepsilon 1}[-]$ - coefficient of the $k-\varepsilon$ model

$C_{\varepsilon 2}[-]$ - coefficient of the $k-\varepsilon$ model

$C_{\mu}[-]$ - coefficient of the $k-\varepsilon$ model

$C_{m}[-]$ - common indication for coefficients of the $k-\varepsilon$ model

$I_{u}[\%]$ - turbulence intensity

$P_{k}\left[\mathrm{~m}^{2} / \mathrm{s}^{3}\right]$ - production of the kinetic turbulence energy

Re [-] - Reynolds number

$k\left[\mathrm{~m}^{2} / \mathrm{s}^{2}\right]-$ kinematic turbulence energy

$p[\mathrm{~Pa}]$ - pressure

$\underline{u}[\mathrm{~m} / \mathrm{s}]-$ velocity vector

$u_{0}[\mathrm{~m} / \mathrm{s}]$ - inlet velocity

$u_{t}[\mathrm{~m} / \mathrm{s}]$ - value of velocity along the wall

$u_{t}^{*}[-]-$ friction velocity

$x_{n}[m]$ - distance between centers of cells adjacent to a wall and this wall

$x_{n}^{*}[-]$ - non-dimensional distance between centers of cells adjacent to walls and these walls

$x_{1}, x_{2}-$ longitudinal and transversal wind tunnel coordinate axes 
$\varepsilon\left[\mathrm{m}^{2} / \mathrm{s}^{3}\right]$ - dissipation of kinematic turbulence energy

$\kappa[-]$ - Kármán's constant

$\mu_{t}\left[\mathrm{Ns} / \mathrm{m}^{2}\right]$ - turbulent dynamic viscosity

$\sigma_{k}[-]$ - coefficient of the $k-\varepsilon$ model

$\sigma_{\varepsilon}[-]$ - coefficient of the $k-\varepsilon$ model

$\rho\left[\mathrm{kg} / \mathrm{m}^{3}\right]$ - air density

Subscripts and Superscripts:

$m$ - reference to coefficients of the $k-\varepsilon$ model

$\sim-$ indication of the sensitivity coefficient of a flow property

$\nabla$ - Nabla operator

\section{Introduction}

The sensitivity analysis serves to check the influence of the small changes of the model parameters on the problem solution. The method is widely applied in the solid mechanics and often used in a design process. Many papers (comp. $[1,2]$ ) describe the usage of sensitivity analysis for evaluation of influence of geometrical parameters or materials parameters on the efficiency of industrial processes. The sensitivity analysis is also applied in the fluid mechanics. This application occurs more rarely, because the problems met in the fluid mechanics are non-linear ones. However, in the last years one can find in the literature (e.g. [3, 4]) the sensitivity analysis as the tool for the design of channels geometry or for research of influence of a body motion in a flow on properties of this flow (pressure, velocity etc.).

In this paper the sensitivity analysis is used to examine how changing the coefficients of the $k-\varepsilon$ model influences on the calculation results of the flow properties. The proposal concept of the use of the sensitivity analysis and led out dependencies have been made by the author herself. The results had been presented in the papers [5-7]. Here are presented the examples of applications of sensitivity analysis, which come from the fact that sensitivity coefficients strongly depend on the approximation degree of flow properties. The problem is presented for three tasks of incompressible steady flow around square and rectangular models with use of the standard and RNG versions of the $k-\varepsilon$ turbulence model.

\section{The sensitivity analysis}

The sensitivity analysis is a method used to examine the influence of changing input parameters on the calculation results. In this paper, the sensitivity analysis is used to examine how changing the coefficients of the $k-\varepsilon$ model influences on the calculation results of the flow properties. For steady incompressible flow there are the following flow properties: pressure $p$, components of velocity vectors $\underline{u}=\left[\begin{array}{lll}u_{1} & u_{2} & u_{3}\end{array}\right]^{\mathrm{T}}$, kinematic turbulence energy $k$, dissipation of kinematic turbulence energy $\varepsilon$ and turbulent dynamic viscosity $\mu_{t}$. These properties are calculated from the system of differential equations which consists of the continuity equation, Navier-Stokes equations, and in the case of the $k-\varepsilon$ turbulence model (comp. [8-10]), the turbulence kinematic energy equation, the dissipation rate of the kinematic turbulence energy equation and equation of the turbulence dynamic viscosity. Since 
these equations contain semi-emprical coefficients (for example, in the standard version of the $k-\varepsilon$ model there are the following coefficients $C_{\varepsilon 1}, C_{\varepsilon 2}, C_{\mu}, \sigma_{k}$ and $\sigma_{\varepsilon}$ ) the calculation results depend on their values. These relationships are described by sensitivity coefficients which may be determined from the system of differential equations obtained after differentiating the equations of the $k-\varepsilon$ model in relation to the parameter marked as $C_{m}$. For the standard version of the $k-\varepsilon$ model this system may be written as (comp. [5-7]):

$$
\begin{aligned}
& \boldsymbol{\nabla} \cdot \tilde{u}_{\mathrm{m}}=0 \\
& \rho\left(\left(\tilde{u}_{\mathrm{m}} \cdot \boldsymbol{\nabla}\right) \mathbf{u}+(u \cdot \nabla) \tilde{u}_{\mathrm{m}}\right)=-\nabla \tilde{p}_{\mathrm{m}}+\tilde{\mu}_{\mathrm{m}} \Delta u+\left(\mu+\mu_{t}\right) \Delta \tilde{u}_{\mathrm{m}} \\
& \rho\left(\left(\tilde{u}_{\mathrm{m}} \cdot \boldsymbol{\nabla}\right) k+(u \cdot \nabla) \tilde{k}_{\mathrm{m}}\right)= \\
& =\left(\boldsymbol{\nabla} \frac{\tilde{\mu}_{\mathrm{m}}}{\sigma_{k}}\right) \cdot(\nabla k)+\frac{\tilde{\mu}_{\mathrm{m}}}{\sigma_{k}} \Delta k+\left(\nabla \frac{\mu_{t}}{\sigma_{k}}\right) \cdot\left(\nabla \tilde{k}_{\mathrm{m}}\right)+\frac{\mu_{t}}{\sigma_{k}} \Delta \tilde{k}_{\mathrm{m}}+\rho \frac{\partial P_{k}}{\partial C_{\mathrm{m}}}-\rho \tilde{\varepsilon}_{\mathrm{m}}+q_{k \mathrm{~m}} \\
& \rho\left(\left(\tilde{u}_{\mathrm{m}} \cdot \boldsymbol{\nabla}\right) \varepsilon+(u \cdot \nabla) \tilde{\varepsilon}_{\mathrm{m}}\right)=\left(\nabla \frac{\tilde{\mu}_{\mathrm{m}}}{\sigma_{\varepsilon}}\right) \cdot(\nabla \varepsilon)+\frac{\tilde{\mu}_{\mathrm{m}}}{\sigma_{\varepsilon}} \Delta \varepsilon+\left(\nabla \frac{\mu_{t}}{\sigma_{\varepsilon}}\right) \cdot\left(\nabla \tilde{\varepsilon}_{\mathrm{m}}\right)+ \\
& +\frac{\mu_{t}}{\sigma_{k}} \Delta \tilde{\varepsilon}_{\mathrm{m}}+\rho C_{\varepsilon 1} \frac{\varepsilon}{k} \frac{\partial P_{k}}{\partial C_{\mathrm{m}}}+\frac{\rho P_{k} C_{\varepsilon 1}-2 \rho \varepsilon}{k} \tilde{\varepsilon}_{\mathrm{m}}-\frac{\rho P_{k} C_{\varepsilon 1} \varepsilon-\rho \varepsilon^{2}}{k^{2}} \tilde{k}_{\mathrm{m}}+q_{\varepsilon \mathrm{m}} \\
& \tilde{\mu}_{\mathrm{m}}-\rho C_{\mu} \frac{2 k}{\varepsilon} \tilde{k}_{\mathrm{m}}+\rho C_{\mu} \frac{k^{2}}{\varepsilon^{2}} \tilde{\varepsilon}_{\mathrm{m}}=q_{\mu \mathrm{m}}
\end{aligned}
$$

where $q_{k m}, q_{\varepsilon m}$ and $q_{\mu m}$ are the terms which are located on the right-hand side and their relationship to each parameter is described with the following formulas:

- for the $C_{\varepsilon 1}$ parameter

$$
q_{\varepsilon 1}=P_{k} \frac{\varepsilon}{k}
$$

- for the $C_{\varepsilon 2}$ parameter

$$
q_{\varepsilon 2}=-\rho \frac{\varepsilon^{2}}{k}
$$

- for the $C_{\mu}$ parameter

$$
q_{\mu 3}=\rho \frac{k^{2}}{\varepsilon}
$$

- for the $\sigma_{k}$ parameter

$$
q_{k 4}=-\left(\nabla \frac{\mu_{t}}{\sigma_{k}^{2}}\right) \cdot(\nabla k)-\frac{\mu_{t}}{\sigma_{k}^{2}} \Delta k
$$

- for the $\sigma_{\varepsilon}$ parameter

$$
q_{\varepsilon 5}=-\left(\nabla \frac{\mu_{t}}{\sigma_{\varepsilon}^{2}}\right) \cdot(\nabla \varepsilon)-\frac{\mu_{t}}{\sigma_{\varepsilon}^{2}} \Delta \varepsilon \text {. }
$$


Sensitivity coefficients may be calculated from the set of Eq. (1-10), but in this paper they are evaluated on the basis of the following formula:

$$
\tilde{s}_{\mathrm{m}}=\frac{w_{2}-w_{1}}{\Delta C_{\mathrm{m}}}
$$

where $w_{1}, w_{2}$ are the results of calculations of the flow problem at $C_{m}-\Delta C_{m} / 2$ and $C_{m}+\Delta C_{m} / 2$, respectively. The discussion of choosing values of $\Delta C_{m}$ and the influence of these values on the results of the sensitivity analysis is presented in the paper [6].

It is shown above that the model coefficients are the factors of derivatives in the set of equations which describes the $k-\varepsilon$ turbulence model. Because of this fact, when the term with the given parameter is large enough, then the value of this parameter, being only the factor, has also the larger influence on the solution. If we analyze problems with the same degree of the approximation of the function, then the larger sensitivity means larger computational errors, because it shows areas with the largest gradients of the flow property function. If we have various orders of approximation of the function, then the largest sensitivity will show us the best of the applied methods of approximation. The more exact approximation usually produces larger values of the derivatives of flow properties and their sensitivity to the parameters of the model. And therefore the results of sensitivity analysis can be applied to:

- show the influence of the $k-\varepsilon$ model parameters on the results of calculations,

- determine the area with the largest errors,

- check quality of solutions and approximation methods,

- research the quality of FVM (Finite Volume Method) meshes.

\section{The numerical analysis}

\subsection{The numerical model}

In order to confirm relationships described above the sensitivity analysis for a few tasks is presented in this paper. To determine the sensitivity coefficients on the basis of Eq. (11) the calculations results obtained from the FLUENT commercial software at two values of the model coefficients are used. The calculations have been made for the following problems:

- model No 1 - the square is located in free flow (Fig. 1), the edge dimension is equal to $D=0.04 \mathrm{~m}$, the inflow velocity is constant $u_{0}=10 \mathrm{~m} / \mathrm{s}$ and the turbulence intensity $I_{u}=0.7 \%$,

- model No 2 - the square is placed at the ground (Fig. 2), the edge dimension is $h_{s}=0.06 \mathrm{~m}$, the turbulence intensity $I_{u}=9.5 \%$ and the inflow velocity is described by the following formulas:

$$
\begin{aligned}
& u_{1}\left(x_{2}\right)=19.7 \mathrm{~m} / \mathrm{s}\left(\frac{x_{2}}{h_{s}}\right)^{0.2} \text { for } x_{2}<0.2 \mathrm{~m}, \\
& u_{1}\left(x_{2}\right)=25 \mathrm{~m} / \mathrm{s} \text { for } x_{2} \geq 0.2 \mathrm{~m},
\end{aligned}
$$


- model No 3 - the rectangle is placed at the ground (Fig. 3), the height of the model is $h_{r}=0.4 \mathrm{~m}$, the width is $a=0.2 \mathrm{~m}$, the turbulence intensity is $I_{u}=6.0 \%$ and the inflow velocity is described by following formulas:

$$
\begin{aligned}
& u_{1}\left(x_{2}\right)=9.67 \mathrm{~m} / \mathrm{s}\left(\frac{x_{2}}{0.5 h_{r}}\right)^{0.15} \text { for } x_{2}<0.25 \mathrm{~m} \text { and } 1.17 \mathrm{~m}<x_{2}<1.42 \mathrm{~m} \\
& u_{1}\left(x_{2}\right)=10 \mathrm{~m} / \mathrm{s} \text { for } 0.25 \mathrm{~m}<x_{2}<1.17 \mathrm{~m} .
\end{aligned}
$$

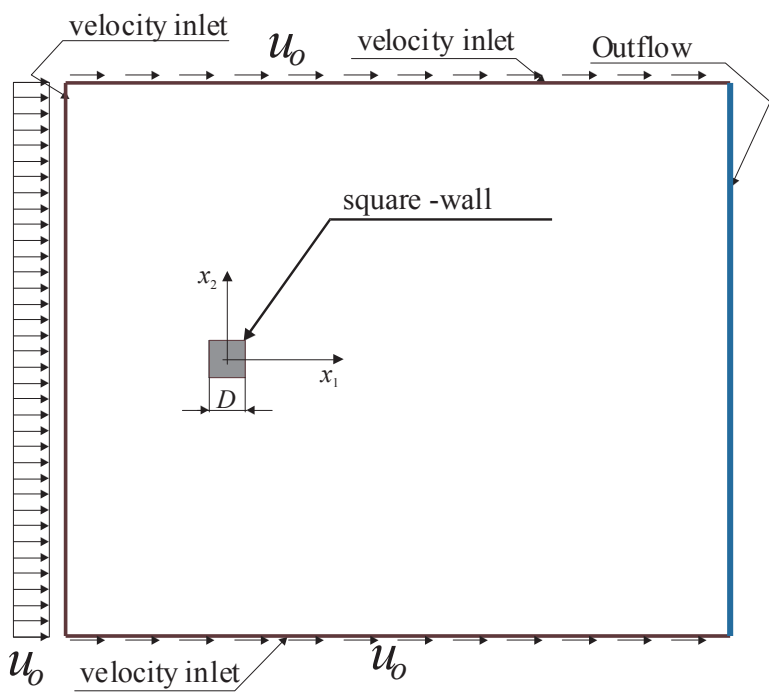

Fig. 1. The computational domain for model No 1.

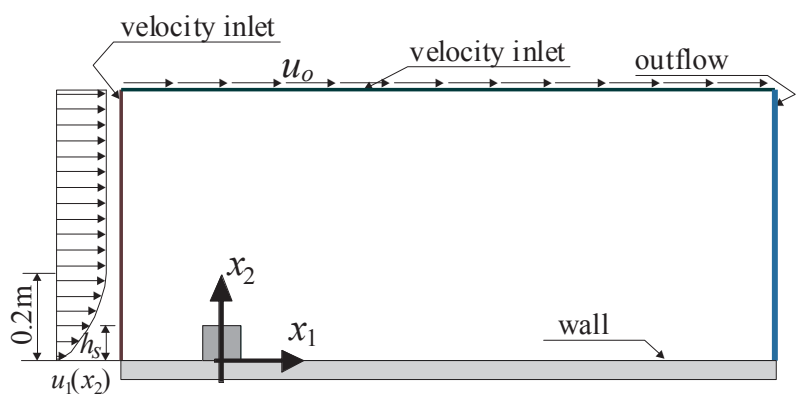

Fig. 2. The computational domain for model No 2.

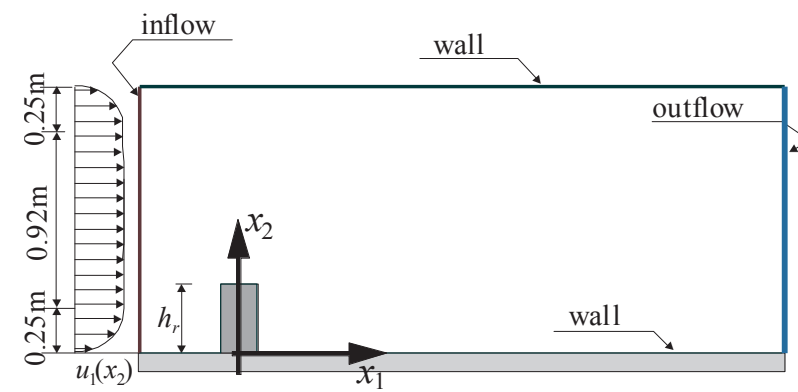

Fig. 3. The computational domain for model No 3. 
The first and the second problems have been calculated by using the standard version of the $k-\varepsilon$ model with the following set of turbulence model coefficients: $C_{\varepsilon 1}=1.44, C_{\varepsilon 2}=1.92, C_{\mu}=0.09, \sigma_{k}=1.0, \sigma_{\varepsilon}=1.3$. For the third task the calculations have been made with use of both the standard and RNG versions. The model coefficients for the RNG version are equal to $C_{\varepsilon 1}=1.42, C_{\varepsilon 2}=1.68, C_{\mu}=0.0845$.

The aim of the paper is to show that the sensitivity coefficients depend on the degree of task approximation. To receive different degrees of approximation for single model, the calculations for the same grid, but different kinematic viscosities have been performed. Thanks to the such formulation of the problem the flows with different gradients are obtained and we are avoiding the impact of differences between solutions caused by, for example, the numbers of iterations or the demanded level of convergence. The second way to obtain the differences in the values of gradients around the walls has been the application of the standard boundary layer model. For this model in the Fluent software the velocity $u_{t}$ along a wall is described by the following formulas ([10-12]):

$$
\begin{aligned}
& u_{t}=\frac{\partial u_{t}}{\partial x_{n}} x_{n} \text { for } x_{n}^{*}<11.225 \\
& u_{t}^{*}=\frac{1}{\kappa} \ln \left(E x_{n}^{*}\right) \text { for } 11.225 \leq x_{n}^{*}
\end{aligned}
$$

The terms from above equations are described by relationships:

- friction velocity

$$
\begin{aligned}
& u_{t}^{*}=x_{n}^{*} \text { for } 11.225>x_{n}^{*} \\
& u_{t}^{*}=\frac{1}{\kappa} \ln \left(E x_{n}^{*}\right) \text { for } 11.225 \leq x_{n}^{*}
\end{aligned}
$$

- non-dimensional distance of centers of cells adjacent to walls

$$
x_{n}^{*}=\frac{\rho C_{\mu}^{1 / 4} k^{1 / 2} x_{n}}{\mu}
$$

The dissipation of the turbulent kinetic energy is calculated from the equation [11]:

$$
\varepsilon=\frac{C_{\mu}^{3 / 4} k^{3 / 2}}{\kappa x_{n}}
$$

This model of the boundary layer is applied in the first row of cells adjacent to walls only, and therefore, non-dimensional distance should be kept in the range $x_{n}^{*} \in(30 ; 60)$. If the distance $x_{n}^{*}$ is smaller than proper one the flow properties are described by wall functions in too narrow areas of calculation domain adjacent to the wall. It causes simplification of the functions of flow properties, and next, significant reduction of the gradients of flow properties. On the other hand, for too big distances $x_{n}^{*}$ and too big sizes of mesh cells the functions describing flow properties are also simplified by using too coarse mesh. It comes from the fact the 
gradients of flow properties are not calculated on the basis of exact derivatives of the function but they are calculated on the basis of numerical methods with correctness dependent on the mesh density. The gradients of the flow functions have significant influence on the sensitivity of flow properties to the turbulence model coefficients, but the sensitivity values also depend on other parameters, the errors of calculations in particularly. Hence, sometimes the similarly high sensitivity is obtained for two tasks: one of these tasks with mesh with the correct non-dimensional distance $x_{n}^{*}$ and the other task with this parameter only close to the proper value. But this fact just confirms the statement that the quality of a FVM mesh around a wall has the most significant influence on the sensitivity coefficients and vice-versa.

Table 1. The $x_{n}^{*}$ value for model No 1 .

\begin{tabular}{cccc}
\hline$R e$ & $\mu$ & $x_{n}^{*}$ & No. of volumes \\
\hline$[/]$ & {$\left[\mathrm{Ns} / \mathrm{m}^{2}\right]$} & {$[/]$} & \\
\hline $2.7 \cdot 10^{2}$ & $1.7894 \cdot 10^{-3}$ & 1.0 & 6440 \\
$2.7 \cdot 10^{3}$ & $1.7894 \cdot 10^{-4}$ & 4.9 & 6440 \\
$2.7 \cdot 10^{4}$ & $1.7894 \cdot 10^{-5}$ & 47.0 & 6440 \\
$2.7 \cdot 10^{5}$ & $1.7894 \cdot 10^{-6}$ & 470.0 & 6440 \\
$2.7 \cdot 10^{6}$ & $1.7894 \cdot 10^{-7}$ & 4700.0 & 6440 \\
\hline
\end{tabular}

Table 2. The $x_{n}^{*}$ value for model No 2 .

\begin{tabular}{cccc}
\hline$R e$ & $\mu$ & $x_{n}^{*}$ & No. of volumes \\
\hline$[/]$ & {$\left[\mathrm{Ns} / \mathrm{m}^{2}\right]$} & {$[/]$} & \\
\hline $1.0 \cdot 10^{2}$ & $1.7894 \cdot 10^{-2}$ & 0.3 & 7830 \\
$1.0 \cdot 10^{3}$ & $1.7894 \cdot 10^{-3}$ & 2.4 & 7830 \\
$1.0 \cdot 10^{4}$ & $1.7894 \cdot 10^{-4}$ & 5.0 & 7830 \\
$1.0 \cdot 10^{5}$ & $1.7894 \cdot 10^{-5}$ & 50 & 7830 \\
$1.0 \cdot 10^{6}$ & $1.7894 \cdot 10^{-6}$ & 490 & 7830 \\
$1.0 \cdot 10^{7}$ & $1.7894 \cdot 10^{-7}$ & 4970 & 7830 \\
\hline
\end{tabular}

Table 3. The $x_{n}^{*}$ value for model No 3 .

\begin{tabular}{ccccc}
\hline$R e$ & $\mu$ & $\begin{array}{c}x_{n}^{*} \\
\text { the standard } \\
\text { version }\end{array}$ & $\begin{array}{c}x_{n}^{*} \\
\text { the RNG } \\
\text { version }\end{array}$ & No. of volumes \\
\hline$[/]$ & {$\left[\mathrm{Ns} / \mathrm{m}^{2}\right]$} & {$[/]$} & {$[/]$} & \\
\hline $2.2 \cdot 10^{2}$ & $1.7894 \cdot 10^{-2}$ & 1.08 & 1.01 & 77275 \\
$2.2 \cdot 10^{3}$ & $1.7894 \cdot 10^{-3}$ & 5.0 & 3.96 & 77275 \\
$2.2 \cdot 10^{4}$ & $1.7894 \cdot 10^{-4}$ & 41.5 & 16.3 & 77275 \\
$2.2 \cdot 10^{5}$ & $1.7894 \cdot 10^{-5}$ & 548.3 & 143.0 & 77275 \\
$2.2 \cdot 10^{6}$ & $1.7894 \cdot 10^{-6}$ & 3369.0 & 1929.8 & 77275 \\
\hline
\end{tabular}

Tables 1, 2 and 3 contain values of the non-dimensional distances $x_{n}^{*}$ for analyzed tasks. The proper values of $x_{n}^{*}$ are bolded in the tables. Since the proper value $x_{n}^{*}$ has not been obtained for the RNG method used for model No 3, the adjacent values are treated as close to proper ones. 


\subsection{The calculation results}

The results of the sensitivity analysis are sensitivity coefficients. The calculation results, which are presented in this paper, have been calculated from Eq. (11) with the following increase of the model coefficients: $\Delta C_{\varepsilon 1}=\Delta C_{\varepsilon 2}=\Delta \sigma_{k}=\Delta \sigma_{\varepsilon}=0.01$ and $\Delta C_{\mu}=0.001$. The numerical calculations have been made with use of the commercial software Fluent. The different values of tolerance of calculation divergence have been applied in each of the tasks.
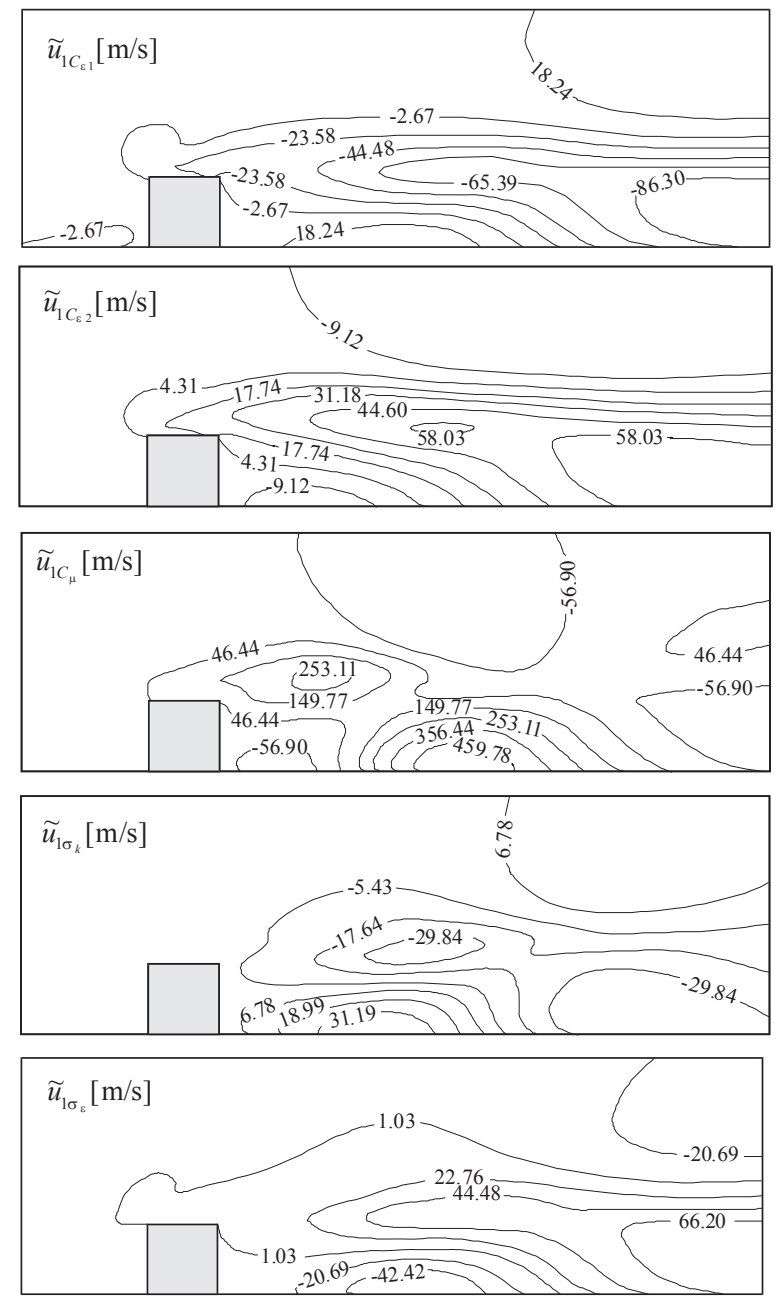

Fig. 4. The fields of the velocity sensitivities for model No 2 .

The tasks for model No 1 and No 2 have been calculated with the best possible tolerance of the calculation convergence. The calculations for model No 3 have been performed at the same tolerance equal to $5 \cdot 10^{-4}$. The exemplary fields of the sensitivity coefficients for model No 2 are shown in Fig. 4. As shown in Figure 4, behind the square, where the vortex is formed and the velocity gradient is large, the velocity sensitivity to the model coefficients is greater than in areas with almost constant values of velocities. 
Figure 5 presents the graphs of velocity sensitivities to all coefficients of the standard $k-\varepsilon$ model behind model No 1 at $x_{1} / D=4.0$. The biggest sensitivity is usually obtained for the Reynolds number equals $10^{3}-10^{4}$. It should be noted that the task with $\operatorname{Re} 10^{4}$ result with the proper value of non-dimensional distance in the boundary layer which gives the proper mesh around the square. The task with $R e \sim 10^{3}$ has similar sensitivity of flow properties to the model coefficients, but as it has been previously explained, sometimes for the non-dimensional distance $x_{n}^{*}$ close to proper value the big values of sensitivity are caused by nearly proper discretization of the problem and by the errors of calculation. Of course, the quality of the mesh has significantly bigger influence than the errors of calculations.
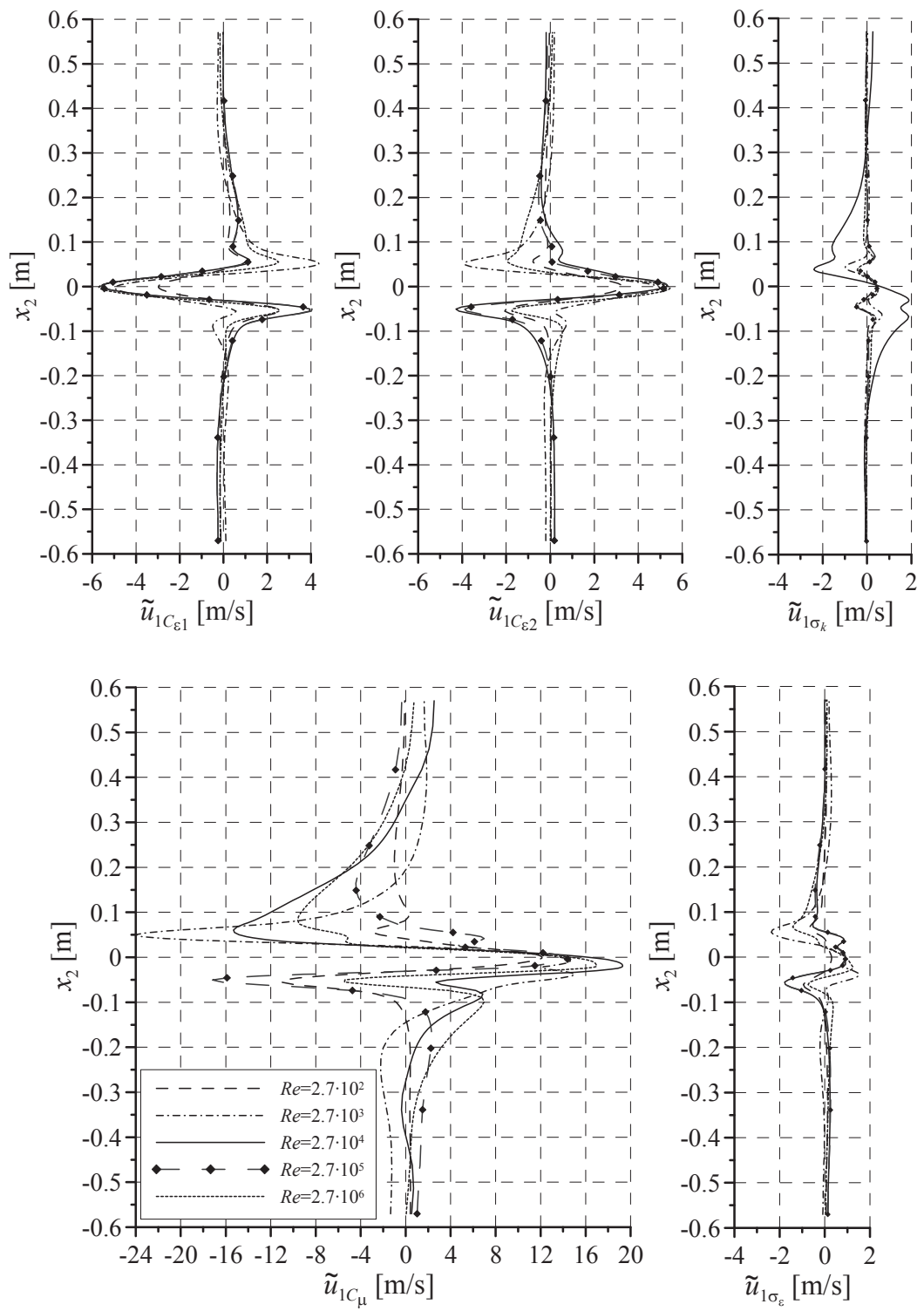

Fig. 5. The graphs of the velocity sensitivities behind model No 1 at $x_{1} / D=4.0$. 

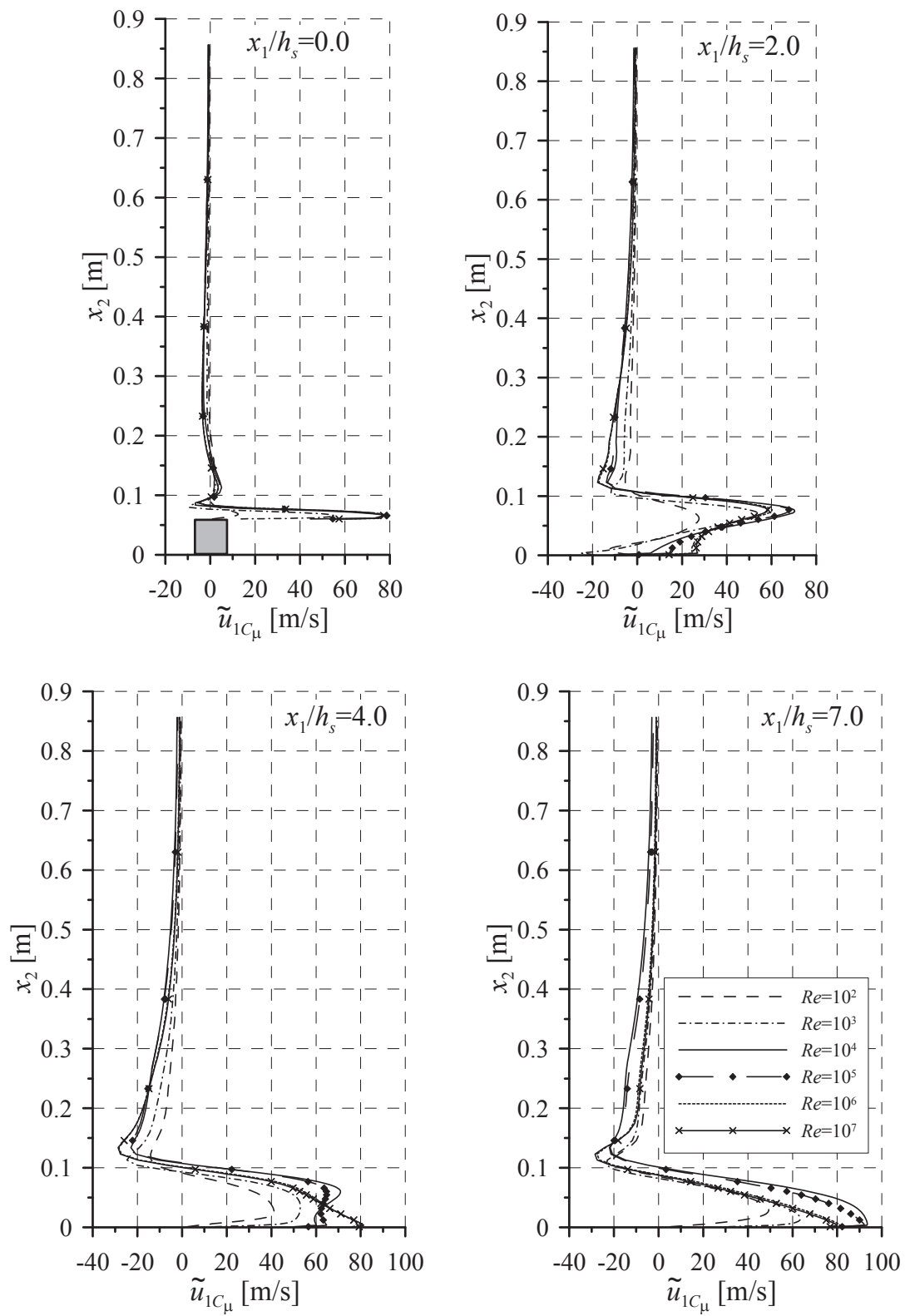

Fig. 6. The exemplary graphs of the velocity sensitivities $\tilde{u}_{1 C_{\mu}}$ around model No 2 . 

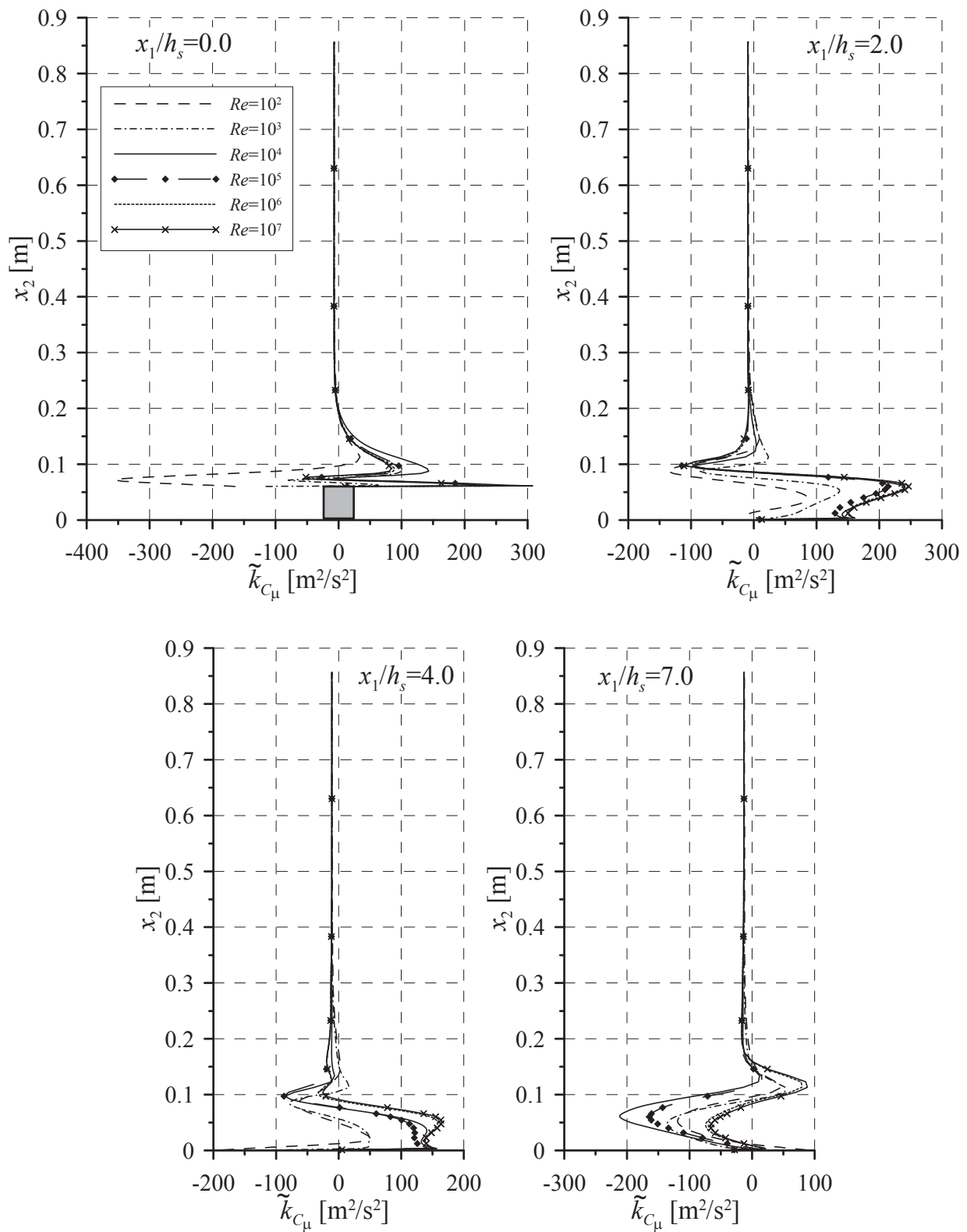

Fig. 7. The exemplary graphs of the turbulence kinetic energy sensitivities $\tilde{k}_{C_{\mu}}$ around model No 2. 

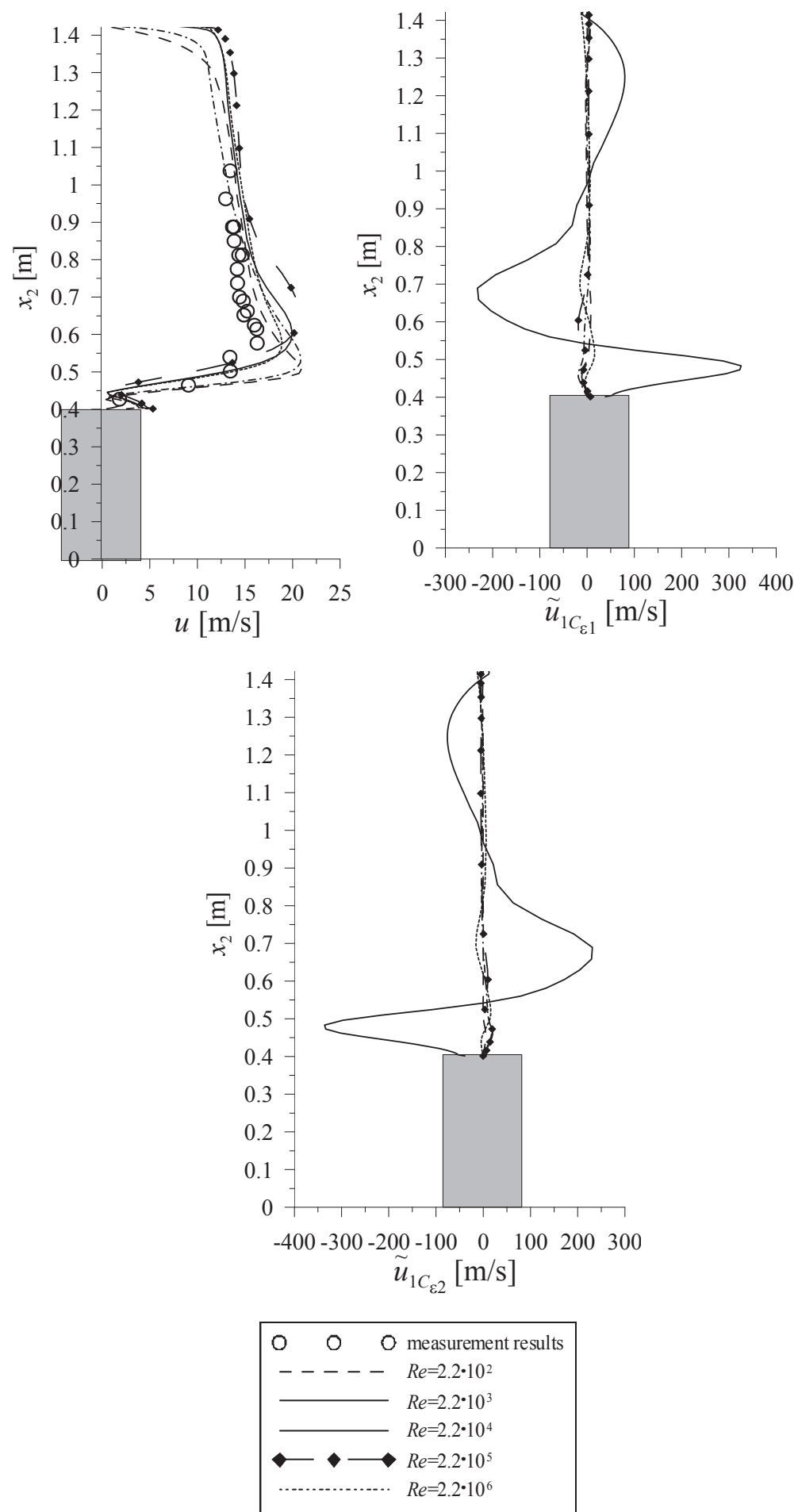

Fig. 8. The graphs of the velocity and its sensitivities to $C_{\varepsilon 1}$ and $C_{\varepsilon 2}$ above model No 3 at $x_{1} / h_{r}=0.0$ obtained from the standard version. 

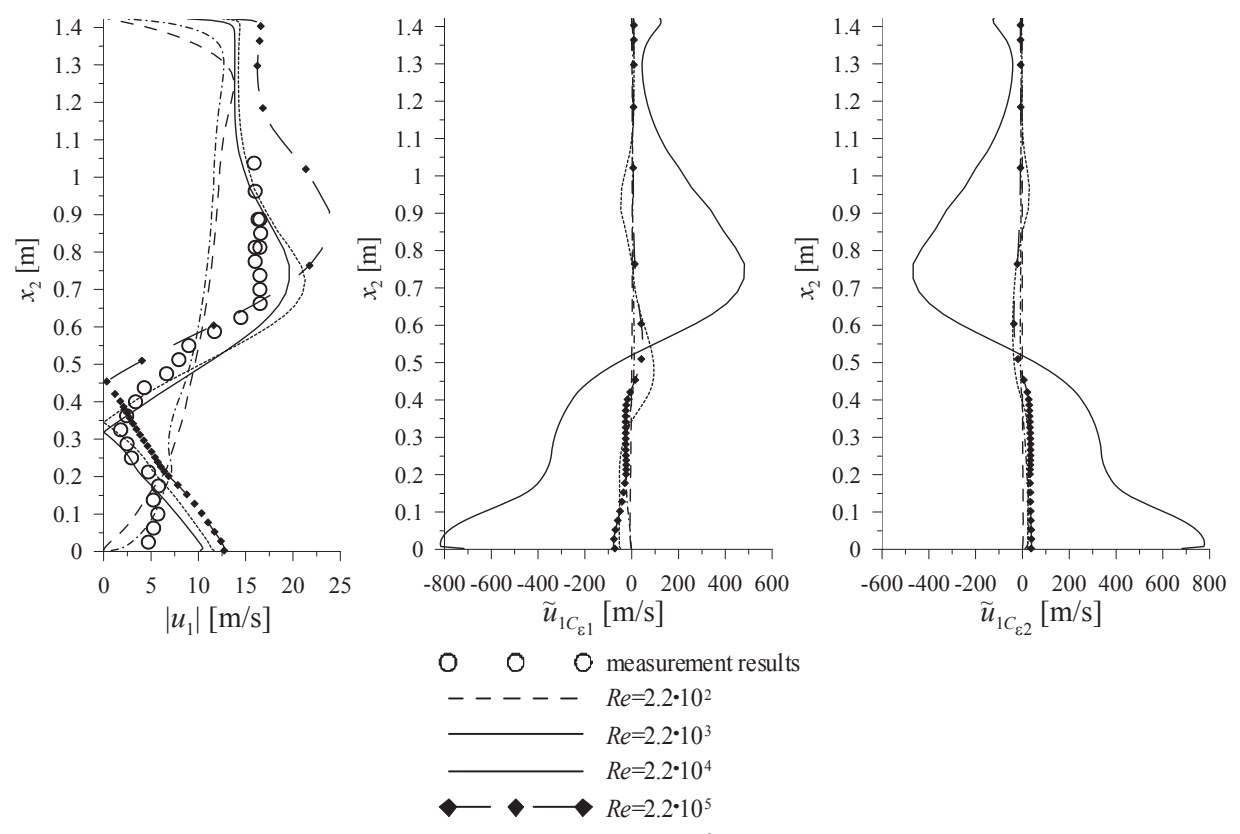

Fig. 9. The graphs of the velocity $u_{1}$ and its sensitivities to $C_{\varepsilon 1}$ and $C_{\varepsilon 2}$ above model No 3 at $x_{1} / h_{r}=2.0$ obtained from the standard version.
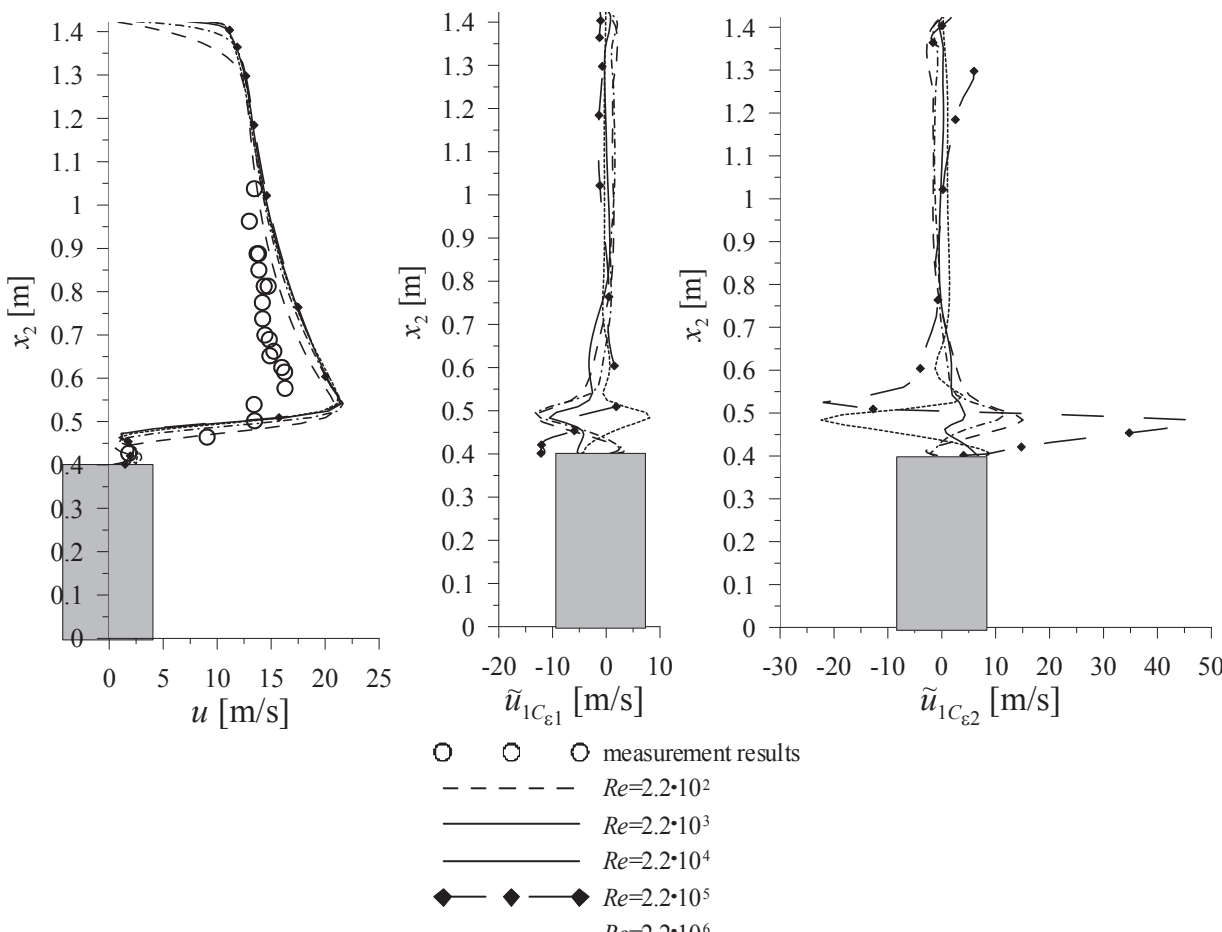

Fig. 10. The graphs of the velocity and its sensitivities to $C_{\varepsilon 1}$ and $C_{\varepsilon 2}$ above model No 3 at $x_{1} / h_{r}=0.0$ obtained from the RNG version. 
Figures 6 and 7 present graphs of the velocity and turbulence kinetic energy as functions of the coefficient $C_{\mu}$ for model No 2. Here, the proper value of nondimensional distance is obtained for Reynolds number equal to $10^{4}$ and $10^{5}$. For this exercise, the tendency of the bigger sensitivity for the better FVM mesh is kept, but in graphs of the sensitivities of turbulent kinematic energy the disturbances of these relationships are visible. The flow parameter sensitivities for Reynolds number bigger than $10^{4}$ are similar, but for the cross sections at $x_{1} / h_{s}=2.0$ the biggest values of the sensitivity are obtained for $R e=10^{7}$.

The sensitivities of flow properties to the model coefficient $C_{\mu}$ do not clearly show the dependence between the quality of the mesh and the sensitivity because this coefficient is not the multiplier of the gradient of flow properties. It is the multiplier at ratio square turbulence kinetic energy and its dissipation. On the other hand the bigger sensitivity for higher Reynolds numbers can be caused by bigger errors of calculations. If the errors are too big the analysis of the quality mesh can be difficult or, for many cases, impossible.

Figures 8-11 show the flow property sensitivities together with the comparison of own measurement at $R e=2 \cdot 10^{5}$ and calculation results for model No 3 . The setup of measurement and their results are described in the papers [13] and [14].
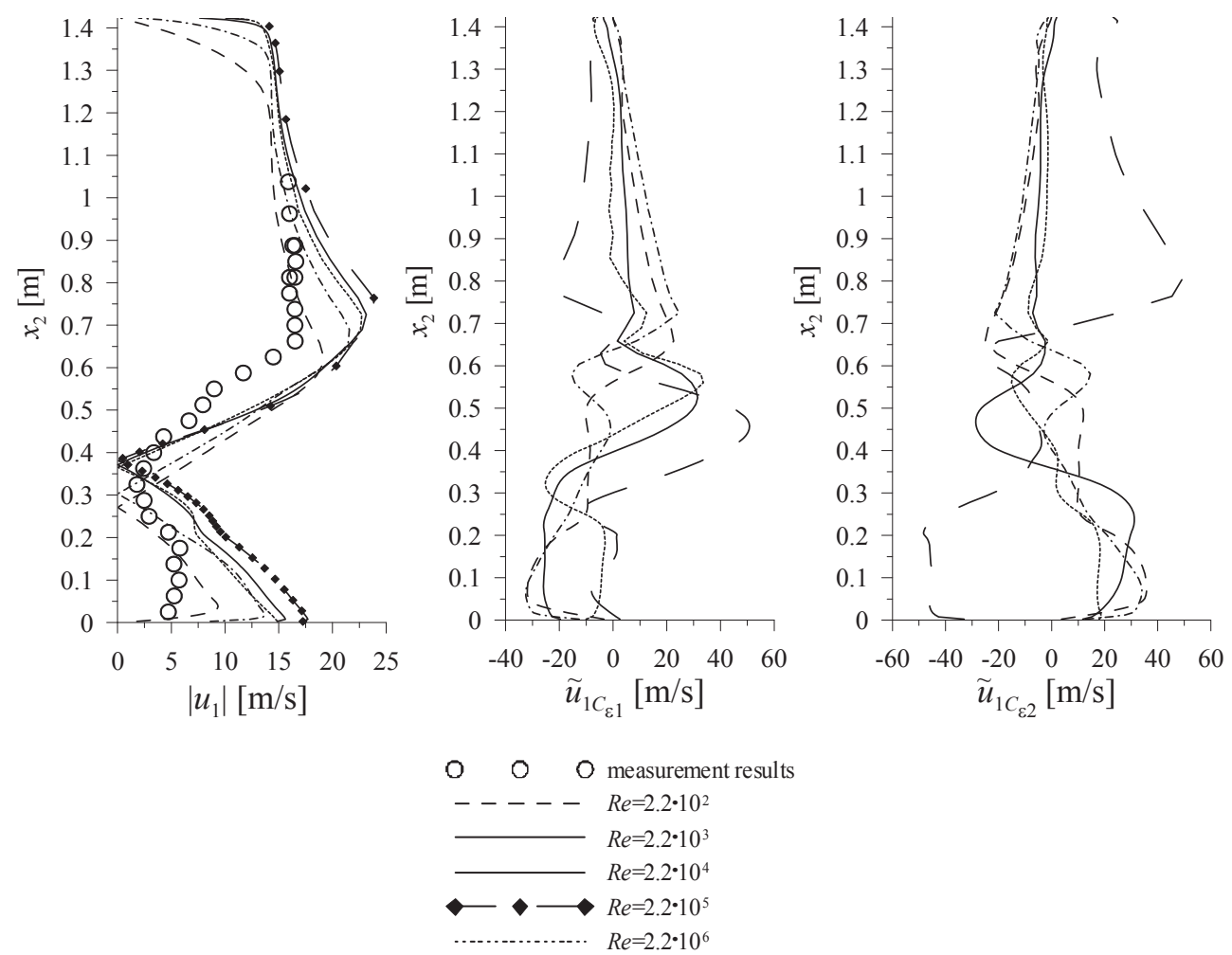

Fig. 11. The graphs of the velocity $u_{1}$ and its sensitivities to $C_{\varepsilon 1}$ and $C_{\varepsilon 2}$ behind model No 3 at $x_{1} / h_{r}=2.0$ obtained from the RNG version of the $k-\varepsilon$ model.

The comparison of flow property sensitivities obtained from standard and RNG versions confirms that the sensitivity is bigger for calculation with bigger 
errors. It is well known that the RNG version gives smaller calculation errors. Here, it is also visible that the results of calculation for the RNG method are closer to measurements than results for the standard version and simultaneously the sensitivity coefficients are smaller for the RNG version. It confirms thesis that at the same approximation of flow properties the bigger sensitivity occurs for bigger errors of calculations.

It is worth remarking that areas of high gradient coincide with areas of high sensitivity. This may be seen at ground and around objects in all figures.

For model No 3 the used meshes are treated as the best for $R e=10^{4}$ for the standard version and close to the best mesh for $R e=10^{4}$ and $R e=10^{5}$ for the RNG method. It comes both from non-dimensional distances of the centers of cells adjacent to walls being proper and from the comparison of calculations and measurements results.

The graphs of sensitivity coefficients shown in Figures 8-11 as in the former figures, keep the tendency of bigger sensitivity for better meshes of calculation domains.

\subsection{The discussion of results}

The simple dependence between Reynolds number and sensitivity cannot be noticed in Figures $5 \div 10$. The change of the sensitivity is connected with the correctness of the flow properties approximation and with the quality of the mesh. The bigger sensitivity means better approximation of the flow properties and it is clearly seen for the components of the velocity. The better approximation of the flow properties is obtained for the better mesh. In other cases the meshes are too coarse at bigger Reynolds numbers or too dense at smaller Reynolds numbers. The sensitivity decreases for more coarse meshes because non-linear functions are „straightened" as the result of the incorrect approximation. Too dense mesh causes the velocity and other flow properties to be described by correct curvilinear functions only in small part of the boundary layer. In other parts they are assumed as constants in cells, and it also reduces the gradients in boundary layer. The variable $x_{n}^{*}$ in the Fluent software is used to calculate the thickness of laminar sublayer near the wall, and so it determines the profile of the velocity function. The one-order growth of the Reynolds number causes the one-order growth of the $x_{n}^{*}$ distance. In the case of too large non-dimensional distance value the viscosity layer of the flow is too wide and in the case of too the small number $x_{n}^{*}$ it is too narrow. In effect velocity values in the boundary layer are incorrectly evaluated.

Each thesis of this paper is confirmed, but in graphs there are also areas with the biggest intensity for the non-proper mesh. The explanation of this situation may be the fact that in calculation domain there are regions with the proper mesh and the regions with the worse one. The next explanation are possibilities to obtain the bigger calculation errors for areas with high gradient of flow properties and hence to obtain bigger sensitivities which are not connected with the level of approximation.

\section{Conclusions}

The paper presents the results of sensitivity analysis of solutions to coefficients of the $k-\varepsilon$ turbulence model and the relationship between the degree of approxima- 
tion of flow properties functions and values of sensitivity coefficients. It seems that this dependence can be useful, for example, to test the quality of the FVM mesh. It is shown that if several meshes of the finite volume method are made and next the sensitivity coefficients are calculated, then for the given Reynolds number the better mesh gives the bigger sensitivities.

\section{References}

[1] Haug H.J., Choi K.K., Komkov V., Design sensitivity analysis of structural systems, Academic Press, Orlando, 1986.

[2] Kleiber M., Antúnez H., Hien T.D., Kowalczyk P., Parameter Sensitivity in Nonlinear Mechanics, John Wiley \& Sons, 1997.

[3] Mohammadi B., Molho J.I., Santiago J. G., Incomplete sensitivities for the design of minimal dispersion fluidic channels, Comput. Methods Appl. Mech. Engineering 192 (2003) 4131-4145.

[4] Fernández M.A., Moubachir M., Sensitivity analysis for an incompressible aeroelastic system, Mathematical Model and Methods in Applied Sciences 12(8) 20021109 1130.

[5] Błazik-Borowa E., The sensitivity analysis of the flow around a square cylinder to parameters of the $k-\varepsilon$ method, The Proc. of the 12th ICWE, Vol. II, 2007, 1919-1926.

[6] Błazik-Borowa E., Analysis of the channel flow sensitivity to the parameters of the $k-\varepsilon$ method", International Journal for Numerical Methods in Fluids 58 (2008) 12571286.

[7] Błazik-Borowa E., The research of the solution quality for the $k$-E turbulence method with using sensitivity analysis of flow properties to model coefficients in Lang P.R., Lombargo F.S. eds. Atmospheric Turbulence, Meteorological Modeling and Aerodynamics, Novapublisher, New York, 2010, pp. 239-276.

[8] Launder B.E., Spalding D.B., Mathematical models of turbulence, Academic Press, London and New York, 1972.

[9] Launder B.E., Spalding D.B., The numerical computation of turbulent flows, Computer Methods in Applied Mech. and Eng. 3 (1974) 269-289.

[10] Johansson A.V., Engineering turbulence models and their development with emphasis on explicit algebraic Reynolds stress models in Oberlack M., Busse F.H. eds. Theories of Turbulence, CISM Courses and Lectures, Springer Vien, New York, 2002, pp. 253-300.

[11] FLUENT 6.1 Documentation, Fluent Inc, 2003.

[12] Schlichting H., Boundary layer theory, McGraw-Hill Book Company, 1979.

[13] Błazik-Borowa E., Bęc J., Nowicki T., Lipecki T., Szulej J., Field measurements of pressure and wind velocity around square and rectangular cylinders, Proc. of 5 th European\&African Conference on wind Engineering, Florencja, 2009, pp. 185-188.

[14] Błazik-Borowa E., Bęc J., Nowicki T., Lipecki T., Szulej J., The measurements of parameters for 2-D flow around square and rectangular cylinders at the ground, Archives of Mechanics and Civil Engineering 11 (2011) 533-551. 